\title{
ANÁLISE DA ACESSIBILIDADE, ACESSOS E CERTIFICAÇÕES DAS INFORMAÇÕES DE UM FÓRUM VIRTUAL DE SAÚDE
}

ANÁLISIS DE ACCESIBILIDAD, ACCESO Y CERTIFICACIONES DE INFORMACIÓN DE UN FORO VIRTUAL DE SALUD

ACCESSIBILITY ANALYSIS, ACCESS AND CERTIFICATIONS OF INFORMATION FROM A VIRTUAL HEALTH FORUM

DOI 10.33194/rper.2019.v2.n2.02.4538 | Submetido 11.12.2018 | Aprovado 03.12.2019

\author{
Michel Marcossi Cintra ${ }^{1}$; ; Naira Favoretto ${ }^{2}{ }^{\circledR}$; Alef Janguas ${ }^{1}{ }^{10}$; Filipe Lopes ${ }^{3}$; \\ Christoph Kaeppler ${ }^{2}$; Fabiana Faleiros ${ }^{1}$ (i⿺
}

1 - Escola de Enfermagem de Ribeirão Preto - Universidade de São Paulo; 2 - Universidade de Dortmund; 3 - Universidade Federal dos Vales do Jequitinhonha e Mucuri (UFVJM), Diamantina

\section{RESUMO}

Introdução: A Espinha Bífida (EB) é a mais frequente malformação congênita no mundo, sendo responsável por importantes sequelas neurológicas, compatível com a vida. Diante disso, faz-se necessário identificar e desenvolver tecnologias educacionais, que auxiliem na capacitação e no apoio a indivíduos com EB e seus familiares. Nessa direção, foi desenvolvido um fórum virtual para indivíduos com EB e seus familiares, o MieloFórum.

Objetivo: A proposta deste estudo é avaliar os acessos, a acessibilidade dos seus usuários e garantir uma certificação de qualidade ao mesmo.

Método: Para a análise de erros de HTML foram coletados os erros de acessos do MieloFórum, analisado os acessos e submetido a uma certificação de qualidade.

Resultados e discussões: o fórum foi desenvolvido e avaliado, sendo encontrado o total de 28 problemas no código do fórum, levando em consideração as regras de padronização do World Wide Web Consortium mostrou-se abrangente em acessos no período analisado e submetido a certificação de qualidade. Foram mais de 1500 acessos em 7 meses com uma demanda em diversos países.

Conclusão: A submissão do MieloFórum em uma ferramenta de análise da acessibilidade mostrou-se útil para o julgamento e a correção dos problemas identificados, potencializando-o juntamente com a certificação de qualidade.

Descritores: Reabilitação,Acesso aos Serviços de Saúde, Fórum de Saúde.

\section{RESUMEN}

Introduccíon: La Espina Bífida (EB) es la más frecuente malformación congénita en el mundo, siendo responsable por importantes secuelas neurológicas, compatible con la vida. Por eso, se hace necesario identificar y desarrollar tecnologías educativas, que ayuden en la capacitación y en el apoyo a individuos con EB y sus familiares. En esa dirección, se desarrolló un foro virtual para individuos con EB y sus familiares, el MieloFórum.

Objetivo: La propuesta de este estudio es evaluar los accesos, la accesibilidad de sus usuarios y garantizar una certificación de calidad al mismo.

Método: Para el análisis de errores de HTML fueron recolectados los errores de accesos de MieloFórum, analizado los accesos y sometido a una certificación de calidad.

Resultados y Discusiones: el foro fue desarrollado y evaluado, encontrando el total de 28 problemas en el código del foro, teniendo en cuenta las nuevas reglas de estandarización del World Wide Web Consortium, se mostró amplio en accesos en el período analizado y sometido a certificación de calidad. Fueron más de 1500 accesos en 7 meses con una demanda en diversos países.

Conclusíon: La sumisión del MieloFórum a una herramienta de análisis de la accesibilidad resultó útil para el juicio y la corrección de los problemas identificados, potenciándolo junto con la certificación de calidad

Palabras clave: rehabilitación, acceso a servicios de salud, foro de salud.

\section{ABSTRACT}

Introduction: Spina Bifida (EB) is the most frequent congenital malformation in the world, responsible for important neurological sequelae, compatible with life. Given this, it is necessary to identify and develop educational technologies, which help in the training and support to individuals with EB and their families. In this direction, a virtual forum was developed for individuals with EB and their relatives, MieloFórum. 
Objective: The purpose of this study is to evaluate the accesses, the accessibility of its users and to guarantee a quality certification to the same.

Method: For the analysis of HTML errors, the access errors of MieloFórum were collected, the accesses analyzed and submitted to a quality certification.

Results and Discussions: the forum was developed and evaluated, and a total of 28 problems were found in the forum code, taking into account the new rules of standardization of the World Wide Web Consortium, it was comprehensive in accesses in the analyzed period and submitted to quality certification . There were more than 1500 accesses in 7 months with a demand in several countries.

Conclusion: MieloForum's submission to an accessibility analysis tool proved to be useful for judging and correcting identified problems, enhancing it together with quality certification.

Keywords: Rehabilitation, Access to Health Services, Health Forum

\section{INTRODUÇÃO}

O MieloFórum foi desenvolvido por grupo de pesquisadores e profissionais da área da saúde de universidades no Brasil e na Alemanha, com experiência na reabilitação de pessoas com Mielomeningocele, onde tiveram a ideia de fazer um fórum virtual para os brasileiros com Mielomeningocele, baseado em um modelo alemão (assim como existe na Alemanha), com o intuito de ajudar as pessoas na troca de informações afim de compartilhar dúvidas, conhecimentos, dificuldades e experiências, criando uma rede de apoio e aprendizagem.

A acessibilidade em ferramentas web refere-se à prática inclusiva de fazer websites que possam ser utilizados por todas as pessoas que tenham deficiência ou não. Quando os sites são corretamente concebidos, desenvolvidos e editados, todos os usuários podem ter igual acesso à informação e funcionalidade. Já o acesso em ferramenta web, refere-se à facilidade de um usuário ir de uma página a outra dentro de sua ferramenta. Há também a interação entre usuários, que se refere à habilidade de se haver uma interação social dentro do fórum e qual sua eficiência (1).

Atualmente a população procura informações sobre saúde na internet. Diante disso há preocupação dos pesquisadores da área da saúde em promover e divulgar informações de saúde de qualidade. Nesse sentido, em 1995, após uma conferência sobre saúde e internet, realizada em Genebra na Suíça, foi criada uma fundação chamada Health on the Net Foundation (HON) fundada para incentivar a divulgação de informações de saúde de qualidade para pacientes, profissionais e públicos em geral, e para facilitar o acesso aos dados de saúde mais recentes por meio da internet. A HON criou um código para estabelecer um padrão ético, com emissão de certificação para websites da área da saúde: o HONcode (2).

A certificação HONcode é um código de ética que visa garantir a divulgação de informações sobre saúde de qualidade. Ele certifica a intenção de um website para publicar informações transparentes e de qualidade. Assim, o HONcode orienta os gestores de sites na criação de um conjunto mínimo de mecanismos para proporcionar qualidade de informação médica objetiva e transparente sob medida às necessidades do público. Sites da área da saúde que solicitam certificação e sites já certificados se comprometem a respeitar o HONcode e os requisitos para a certificação.
A posse de selo HONcode permite um site para demonstrar a sua intenção de contribuir para a informação de saúde de qualidade através da publicação de informação objetiva e transparente ${ }^{(2)}$.

Para receber a certificação é necessário o cumprimento dos 8 princípios do HONcode, são eles:

Princípio 1 - Autoridade: toda orientação médica ou de saúde hospedado e prestado no site será dada somente por profissionais treinados e qualificados, a menos que uma declaração clara de que o conselho oferecido é de um indivíduo não qualificado na área médica ou organização.

Princípio 2 - Complementaridade: as informações fornecidas neste site destinam-se a apoiar, e não substituir, a relação que existe entre um paciente/visitante do site e o seu/sua médico (a) existente.

Princípio 3 - Confidencialidade: privacidade dos dados relacionado aos pacientes e visitantes individuais a um site médico/saúde, incluindo a sua identidade, é respeitada por este site. Os responsáveis pelo site se comprometem em honrar ou exceder os requisitos legais de informação médica/saúde privacidade que se aplicam no país e no estado onde os Website e seus espelhos estão localizados.

Princípio 4 - Atribuição: se for o caso, a informação contida no site será respaldada por referências claras às fontes consultadas, e, quando possível, tendo links HTML para estas fontes. A data em que cada página médica foi modificada pela última vez será exibida claramente (por exemplo, na parte inferior da página).

Princípio 5 - Justificativas: quaisquer afirmações feitas sobre os benefícios e / desempenho de um tratamento específico, produto ou serviço comercial será apoiada por evidência adequada, equilibrada, conforme indicado no Princípio 4.

Princípio 6 - Transparência: os designers do site irão procurar dispor a informação da forma mais clara possível e disponibilizar endereços de contato para os visitantes que desejem informação ou ajuda adicional. 0 webmaster exibirá seu endereço de e-mail claramente em todo o Web site.

Princípio 7 - Divulgação financeira: contribuição para este site serão identificados claramente, incluindo a identidade das organizações comerciais e não- 
comerciais que tenham contribuído através de financiamento, serviços ou material para o site.

Princípio 8 - Publicidade: se a publicidade é uma fonte de financiamento, isto deverá ser indicado claramente. Uma breve descrição da política de divulgação adotada pelos proprietários do Web site será exibido no site. Os anúncios e outros materiais promocionais serão apresentados aos visitantes de uma maneira e contexto que facilitem diferenciá-los do material original produzido pela instituição gestora do site.

Para plataformas colaborativas online, como é o caso de fórum virtual, além dos 8 princípios citados acima, a privacidade e as mensagens também são avaliadas.

$\mathrm{O}$ desenvolvimento de uma ferramenta que permita a divulgação de informações sobre saúde de qualidade e a troca de experiências entre os usuários, como um fórum virtual, traz a responsabilidade de certificar de que essas informações sejam passadas com qualidade para a real intenção de servir de apoio mutuo a população, com isso este estudo visa analisar acessos, acessibilidade e certificar as informações de um fórum virtual de saúde (MieloFórum).

\section{MÉTODO}

Este estudo, trata-se de uma pesquisa descritiva. Por ser um projeto com dados de livre acesso e sem possibilidade de identificação dos participantes dispensando assim, a aprovação pelo comitê de ética em pesquisa.

A checagem da acessibilidade foi realizada com o uso do Web Accessibility Checker, ferramenta de avaliação web que ajuda desenvolvedores a avaliarem o códigofonte de seus websites ${ }^{(1)}$. A AChecker utiliza as normas padrões detalhadas no World Wide Web Consortium, principal organização internacional de normalização para World Wide Web (WWW, rede mundial de computadores) ${ }^{(3)}$ com o AChecker foi realizada uma varredura no código-fonte, página por página do MieloFórum, verificando os problemas e os potencias problemas. Após essa varredura a ferramenta emitiu um relatório com a identificação dos problemas e potenciais problemas. Os dados foram organizados e analisados com o auxílio de linguagem de programação PHP (Hypertext Preprocessor) e do programa Excel.

A HON recomenda que a qualidade dos sites deva ser certificada por uma organização neutra baseada em critérios de qualidade, como o HONcode. (6) A certificação do MieloFórum pela Health on the Net Foundation foi realizada após a submissão do mesmo ao Health on The Net Code no site da HON e obtenção do selo de certificação, com reavaliação anual. Os processos de certificação e reavaliação encontram-se elucidados na figura1.

Os indicadores web permitem medir e avaliar atividades científicas e técnica ,onde diversos indicadores podem ser construídos e analisados, como, por exemplo: tamanho ou número de objetos de um espaço web em países, regiões, organizações, pessoas, quanto ao conteúdo; exames das conexões entre páginas e sites; quantidades e características das visitas que cada site recebe; densidade da rede; número de visitas recebidas por um site; número de links recebidos ${ }^{(7)}$. Para a análise estatística foi utilizado a ferramenta Google analytics, o qual é uma ferramenta gratuita que permite obter estatísticas de um site ou loja virtual para, assim, compreender o comportamento dos usuários, o site foi analisado do período de disponibilização ao público 28 de novembro de 2014 ao dia 30 de junho de 2015.

\section{RESULTADOS}

Desenvolvimento do fórum virtual: o fórum foi construído no sistema PhpBB (sistema gerenciador de fóruns para a Internet), com a utilização de scripts em PHP, assim como o fórum alemão colaborador deste estudo. Foi utilizado uma versão customizada do style, conjunto de grupo de imagens, templates e temas, coffe time. A customização foi realizada com o objetivo de se trazer maior facilidade de navegação e entendimento para os usuários do fórum.

A customização passou pelas seguintes etapas: personalização de botões e abas, escolha de cores e inclusão de MODs (modificações ao fórum). Ressaltando a constante atualização do fórum, os MODs adicionados até o momento foram: botão Private_Message_Moderation, utilizado para haver o controle de mensagem privada entre usuários do fórum, e FAQ Manager, utilizado para facilitar as alterações na página de perguntas mais frequentes.

Juntamente com as modificações começaram a ser realizadas as inclusões de material teórico sobre a temática do fórum (Mielomeningocele, cateterismo vesical intermitente, entre outros), considerando a dinâmica e facilidade da linguagem para o público alvo.

Para a análise da acessibilidade foi utilizada a ferramenta Web Accessibility Checker (AChecker). Esta ferramenta verifica páginas HTML individuais para conformidade com os padrões de acessibilidade para garantir que o conteúdo pode ser acessado por todos.

Após a análise foram encontrados 28 problemas com o código do fórum, conforme a tabela 1.

A seguir serão descritos os problemas identificados em cada página, seguidos das análises e correções realizadas:

\section{Página 1: foram encontrados sete problemas com o} código do fórum:

Do primeiro ao sexto foram problemas com o código $<\mathrm{b}\rangle$ (negrito em html). O Web Accessibility Checker atualmente utiliza as bases de HTML5 para avaliação de acessibilidade, ou seja, está alegando que deveria ser modificado no código-fonte, todos os $\langle\mathrm{b}\rangle$ (negrito em html) por <strong>(negrito em HTML5). No entanto, como estamos utilizando uma ferramenta, phpBB, que é feita para facilitar tanto a navegação dos usuários quanto a possíveis modificações no fórum através de futuros administradores e moderadores, o ideal é manter o padrão da ferramenta phpBB. Dessa maneira, após a análise, não foram necessárias correções para esses 6 problemas identificados. 

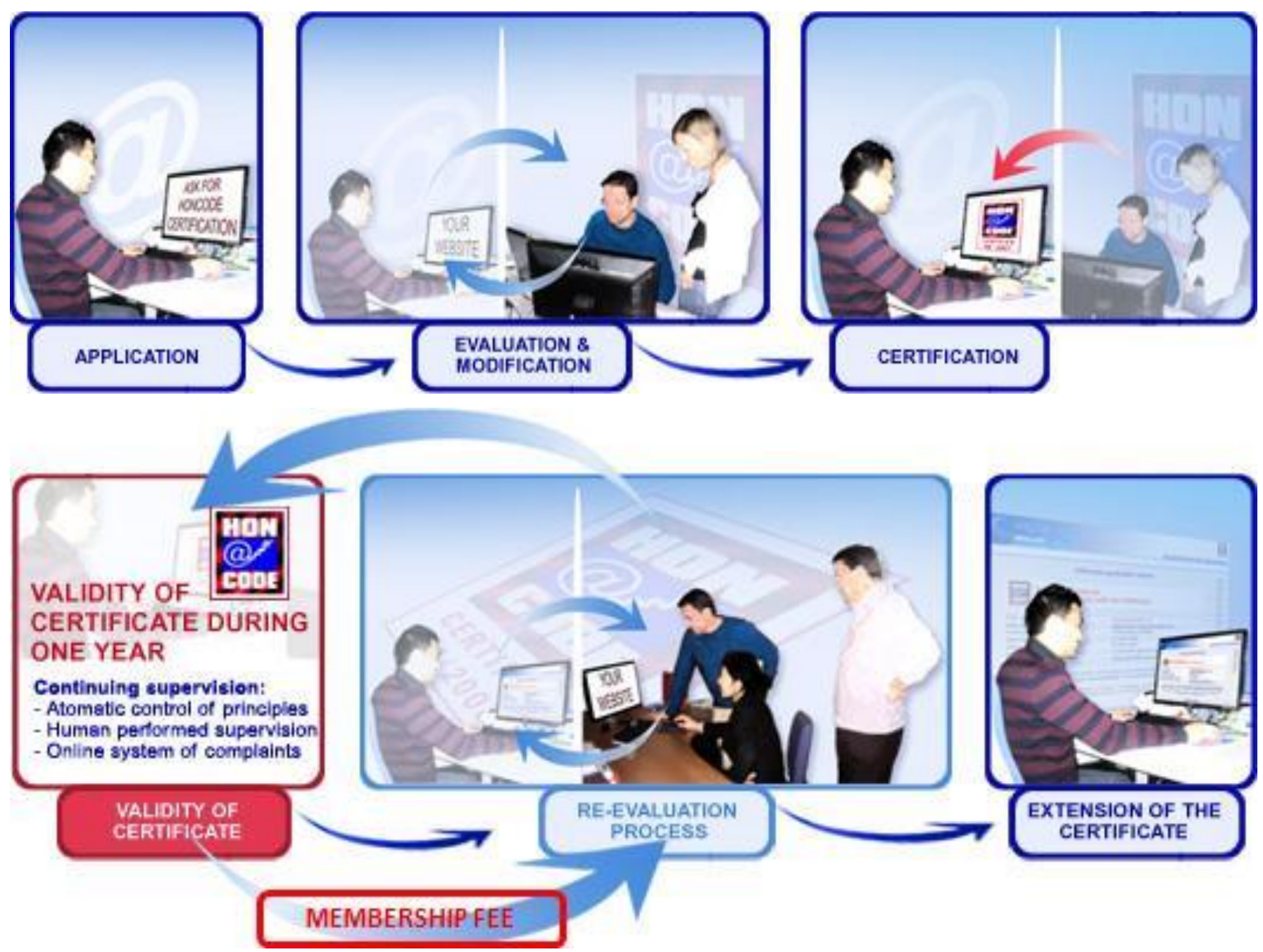

Figura 1 - Processo de Certificação HON

\begin{tabular}{|l|c|}
\hline Número e Nome da Página & Problemas \\
\hline 1. Índice do fórum & 7 \\
\hline 2. Entrar & 0 \\
\hline 3. Registro & 0 \\
\hline 4. Questões mais frequentes & 5 \\
\hline 5. Seja bem-vindo ao “MieloFórum” & 0 \\
\hline 6. MieloFórum/Discussões & 0 \\
\hline 7. Informações sobre o MieloFórum & 8 \\
\hline 8. Termos de uso MieloFórum & 0 \\
\hline 9. Quem Somos & 0 \\
\hline 10. Contato & 0 \\
\hline 11. Saiba mais sobre a Mielomeningocele & 8 \\
\hline 12. O que é Espinha Bífida? & 0 \\
\hline 13. O que é Bexiga Neurogênica? & 0 \\
\hline 14. Cateterismo Vesical Intermitente & 0 \\
\hline $\begin{array}{l}\text { 15. Infecção urinária e cateterismo vesical } \\
\text { intermitente }\end{array}$ & 0 \\
\hline 15 páginas & 28 \\
\hline
\end{tabular}

Fonte: AChecker

Tabela 1 - Distribuição das páginas do MieloFórum segundo os problemas identificados na avaliação do AChecker. Pesquisa realizada em ambiente virtual, 2014
0 sétimo problema foi com o código <h1> (encabeçamento ou título em html) alegando que ele estaria vazio. Após analisar, foi encontrado que $0<\mathrm{h} 1\rangle$ é uma das possíveis modificações que podem ser feitas através de uma ferramenta facilitadora do phpBB o ACP (Administration Control Panel). Assim seria necessária uma correção, no entanto optamos por utilizar o logotipo do fórum como o título. Além disso, como o fórum pode vir a ser modificado no futuro, para atualizações e adaptações ao seu público, retirar os códigos $<\mathrm{h} 1>$, seria retirar essa utilidade da nossa ferramenta.

Página 4: foram encontrados cinco problemas com o código do fórum:

Do primeiro ao quinto foram problemas com o código <i> (itálico em html). O Web Accessibility Checker atualmente utiliza as bases de HTML5 para avaliação de acessibilidade, ou seja, está alegando que deveria ser modificado no código-fonte, todos os $<i>$ (itálico em html) por <em> (negrito em HTML5). Mas como estamos utilizando uma ferramenta, phpBB, que é feita para facilitar tanto a navegação dos usuários quanto a possíveis modificações no fórum através de futuros administradores e moderadores, optamos por manter o padrão da ferramenta phpBB e não realizar modificações. 


\section{Página 7 e 11: foram encontrados oito problemas com o código do fórum:}

Do primeiro ao oitavo foram problemas com o código $\langle\mathrm{b}\rangle$ (negrito em html). O Web Accessibility Checker atualmente utiliza as bases de HTML5 para avaliação de acessibilidade, ou seja, está alegando que deveria ser modificado no código-fonte, todos os $\langle\mathrm{b}\rangle$ (negrito em html) por <strong>(negrito em HTML5). Mas como estamos utilizando uma ferramenta, phpBB, que é feita para facilitar tanto a navegação dos usuários quanto a possíveis modificações no fórum através de futuros administradores e moderadores, optamos por manter 0 padrão da ferramenta phpBB e não realizar modificações.

\section{Páginas 2,3,5,6,8,9,10,12,13,14 e 15:}

Nenhum problema foi encontrado.

O AChecker salientou também o possível problema com a nomeação dos nossos objetos no CSS (Cascading Style Sheets - uma linguagem de folhas de estilo utilizada para definir a apresentação de documentos escritos em uma linguagem de marcação, como HTML), alegando que os objetos possuem títulos irrelevantes, no entanto esses títulos são utilizados pelo ACP (Painel de Controle do Administrador) para fornecer uma forma facilitada de se alterar o fórum pelos administradores.

Certificação do MieloFórum pelo HONcode: o fórum foi submetido à certificação do HONcode, a iniciativa da Fundação Health On the Net para melhorar a qualidade da informação médica na Internet. 0 website foi visto e avaliado relativamente ao cumprimento dos princípios do HONcode e as seguintes modificações/alterações precisaram ser realizadas:

Princípio 3 - Confidencialidade - Criação de uma descrição ou uma página contendo a política de privacidade dos dados recolhidos pelo website. Foi criada uma página no fórum com o nome: "Termos de uso MieloFórum" com toda a política de confidencialidade e uso do fórum.

Princípio 7 - Fonte de Financiamento - Como é o website financiado? - Indicar de forma clara como é que o seu website é financiado. Foi elaborada uma página no fórum com o nome: "Parceiros" com todos os financiamentos ao MieloFórum.

Princípio 8 - Honestidade na publicidade e política editorial - Deve ser claramente mencionado que o website não possui links ou banners publicitários. Foi adicionado na página "Parceiros" a frase: "O MieloFórum atualmente não possui qualquer forma de publicidade".

Princípios Health 2.0 - Se aplicam a plataformas colaborativas online, como por exemplo, fóruns virtuais. Seguindo esse princípio foi adicionado aos "Termos de uso MieloFórum", mais quatro itens oferecendo esclarecimento ao usuário em relação à privacidade, mensagens, complementariedade e autoridade do MieloFórum. Foram realizadas todas as correções sugeridas pelos certificadores da HON e reenviado o pedido de certificação e concedido no mês de março.
Os dados de acessos/sessões do MieloFórum foram categorizados conforme a tabela 2.

\begin{tabular}{|l|c|}
\hline Acessos & 1548 \\
\hline Usuários & 938 \\
\hline Visualizações de Página & 8574 \\
\hline Taxa de Rejeição & $57,04 \%$ \\
\hline Porcentagem de novas sessões & $60,34 \%$ \\
\hline Página/Sessão & $5,54 \%$ \\
\hline
\end{tabular}

Fonte: Google Analytics

Tabela 2: Dados de acessos/sessões do MieloFórum. Pesquisa realizada em ambiente virtual, 2014

Observamos na Tabela 2 que o MieloFórum obteve em seu período de análise 1.548 sessões, com 938 usuários, 8.574 visualizações de páginas foi observada ainda uma taxa média de rejeição de $57,04 \%$, que significa que o novo usuário ficou conectado na página do MieloFórum por menos de 3 minutos. $60,34 \%$ de novas sessões e uma média de 5,54 páginas por sessão.

A distribuição das sessões no MieloFórum está categorizada de acordo com o número e da procedência (país) dos acessos, conforme a tabela 3.

\begin{tabular}{|l|c|c|}
\hline \multicolumn{1}{|c|}{ País } & Acessos & $\%$ \\
\hline Brasil & 671 & 43 \\
\hline Alemanha & 293 & 18,93 \\
\hline Rússia & 133 & 8,59 \\
\hline Estados unidos & 126 & 8,14 \\
\hline China & 123 & 1,94 \\
\hline Portugal & 30 & 1,61 \\
\hline Japão & 25 & 1,16 \\
\hline Coréia do Sul & 18 & 0,78 \\
\hline Holanda & 12 & 0,78 \\
\hline İndia & 12 & 0,71 \\
\hline Canadá & 11 & 0,52 \\
\hline Inglaterra & 8 & 0,52 \\
\hline Angola & 8 & 0,39 \\
\hline Taiwan & 6 & 0,39 \\
\hline Itália & 6 & 0,32 \\
\hline Outros Países & 5 & 3,41 \\
\hline Not Set & 61 & 7,95 \\
\hline Total de Acessos & 1548 & 100 \\
\hline
\end{tabular}

Fonte: Google Analytics

Tabela 3: Distribuição das sessões ao MieloFórum de acordo com o país de acesso. Pesquisa realizada em ambiente virtual, 2015

Observa-se na Tabela 3 que o Brasil lidera o número de acessos ao MieloFórum como esperado com $43 \%$ dos acessos, foram 7,95\% o número de acessos de computadores não identificados pelo Google analitycs pelo seu país de origem, Alemanha obteve $18,93 \%$ dos acessos seguido por Rússia $(8,59 \%)$, Estados Unidos $(8,14 \%)$, China $(1,94 \%)$, Portugal $(1,61 \%)$, Japão $(1,16 \%)$, Coréia do Sul $(0,78 \%)$, Holanda $(0,78 \%)$, Índia $(0,71 \%)$, Canadá $(0,52 \%)$, Inglaterra $(0,52 \%)$, Angola $(0,39 \%)$, Taiwan $(0,39 \%)$, Itália $(0,32 \%)$ e outros países 
com 2 acessos ou menos somaram $(3,41 \%)$ de acessos ao MieloFórum.

A palavra "not set" exibida nas tabelas do Google Analytics mais abaixo, significa que o website recebe tráfego de uma conta do Google AdWords que não está vinculada à visualização de relatórios,_ou seja, o IP não é reconhecido pelo sistema.

A distribuição das sessões no MieloFórum provenientes do Brasil está categorizada de acordo com o número dos acessos e o correspondente estado, conforme a tabela 4.

\begin{tabular}{|l|c|c|}
\hline \multicolumn{1}{|c|}{ Estados } & Acessos & $\%$ \\
\hline São Paulo & 251 & 37,41 \\
\hline Minas Gerais & 86 & 12,82 \\
\hline Paraná & 64 & 9,54 \\
\hline Rio de Janeiro & 63 & 9,39 \\
\hline Santa Catarina & 33 & 4,92 \\
\hline Rio Grande do Sul & 22 & 3,28 \\
\hline Distrito Federal & 15 & 2,24 \\
\hline Piauí & 15 & 2,24 \\
\hline Goias & 14 & 2,09 \\
\hline Para & 14 & 2,09 \\
\hline Bahia & 12 & 1,79 \\
\hline Ceará & 12 & 1,79 \\
\hline Mato Grosso do Sul & 11 & 1,64 \\
\hline Pernambuco & 11 & 1,64 \\
\hline Maranhão & 7 & 1,04 \\
\hline Paraíba & 7 & 1,04 \\
\hline Rio Grande do Norte & 6 & 0,89 \\
\hline Mato Grosso & 5 & 0,75 \\
\hline Amazonas & 4 & 0,60 \\
\hline Espírito Santo & 4 & 0,60 \\
\hline Amapá & 4 & 0,60 \\
\hline Sergipe & 3 & 0,45 \\
\hline Roraima & 1 & 0,15 \\
\hline Tocantins & 1 & 0,15 \\
\hline Acre & 1 & 0,15 \\
\hline Not Set & 671 & 0,75 \\
\hline Total & 100 \\
\hline
\end{tabular}

Fonte: Google Analytics

Tabela 4 - Distribuição das sessões ao MieloFórum no Brasil de acordo com o estado de acesso. Pesquisa realizada em ambiente virtual,2015.

Conforme a tabela 4, São Paulo é o estado com maior número de acessos ao MieloFórum com 37,41\% dos acessos, seguido por Minas Gerais $(12,82 \%)$, Parana $(9,54 \%)$, Rio de Janeiro $(9,39 \%)$, Santa Catarina $(4,92 \%)$, Rio Grande do Sul (3,28\%), Distrito federal (2,24\%), Piaui (2,24\%), Goias (2,09\%), Pará $(2,09 \%)$, Bahia $(1,79 \%)$, Ceará $(1,79 \%)$, Mato Grosso do Sul $(1,64 \%)$, Pernambuco (1,54\%), Maranhão (1,04\%), Paraíba $(1,04 \%)$, Rio Grande do Norte $(0,89 \%)$ Not Set $(0,75 \%)$, Mato Grosso $(0,75 \%)$, Amazonas $(0,60 \%)$, Espirito Santo
$(0,60 \%)$, Amapá $(0,60 \%)$, Sergipe $(0,45 \%)$, Roraima $(0,15 \%)$, Tocantins $(0,15 \%)$, Acre $(0,15 \%)$.

A maioria dos acessos foram oriundos de computadores $(79,97 \%)$, enquanto $18,48 \%$ dos acessos foram a partir de celulares e $1,55 \%$ por tablets.

\section{DISCUSSÃO}

O desenvolvimento de uma ferramenta virtual em saúde que busca ser acessível e adequada, tanto às pessoas com MMC quanto aos seus familiares, favorece o acesso às informações sobre a malformação e a troca de experiência entre os usuários, aumentando o conhecimento e permitindo a formação de uma rede de apoio mútuo. Nesse sentido, é importante considerar que além de ser incentivado à população e aos profissionais o uso de tecnologias, deve-se considerar os familiares/ cuidadores e incluir a sua participação ${ }^{(8)}$.

O sistema gerenciador de fóruns para a Internet que está sendo utilizado, o phpBB, é uma plataforma antiga e que possui alguns problemas com as novas padronizações do WW3 e consequentemente do AChecker. O style utilizado foi desenvolvido pela comunidade do phpBB, no entanto, não foram permitidas todas as alterações desejadas durante o desenvolvimento do fórum, uma vez que o suporte da plataforma é limitado.

A internet apresenta um conteúdo extenso de informações sobre saúde disponibilizada a uma população diversificada, as quais podem apresentar as mais diferentes habilidades e exigências ${ }^{(4,9)}$ Além da busca por informações, as pessoas que buscam informações, desejam compartilhar suas aflições e experiências $^{(10)}$.

Dessa maneira, garantir a acessibilidade a todo usuário é um desafio, mesmo existindo um conjunto extenso de recomendações disponibilizadas pelo World Wide Web Consortium $^{(3)}$. Assim, são propostas diferentes ferramentas para avaliação de acessibilidade, que contrapõem os artefatos às diretrizes com a finalidade de obter resultados automatizados, produzindo testes e gerando vários dados, como a localização do problema no código e as falhas especificadas (4) Para facilitar o processamento desses dados, mediante a disponibilização de uma linguagem comum, foi utilizado o AChecker para rever a acessibilidade das páginas Web com base em uma variedade de diretrizes internacionais de acessibilidade. De acordo com os resultados, observou-se que a plataforma PhpBB utilizada não permitiu todas as alterações indicadas pelo AChecker e, portanto, seria recomendado transportar o MieloFórum para uma plataforma mais moderna.

O reconhecimento do papel central da Internet como fonte de informação sobre saúde, tem gerado uma crescente preocupação com a qualidade e a transparência dessas informações obtidas pela população ${ }^{(11-12)}$. Em resposta a essas preocupações, foram desenvolvidas uma série de iniciativas para estabelecer critérios de qualidade para sites relacionados com a saúde e para ajudar os usuários a encontrar informações de qualidade. Nessa direção foi 
realizada submissão do MieloFórum, a rotulagem de qualidade, realizada pela Health on the Net Foundation (HON), com foco em padrões éticos relacionados à publicação on-line. Uma vez que a HON recomenda que a qualidade dos sites deve ser certificada por uma terceira e neutra organização, de acordo com critérios de qualidade, como o HONcode ${ }^{(2,4)}$

A certificação do MieloFórum pelo HONcode foi extremamente produtiva, pois permitiu modificações na ferramenta, essenciais para a adequação da mesma, elevando-a a uma padronização internacional. Cabe ressaltar que a mesma preocupação em certificar ferramentas virtuais foi demonstrada por outros pesquisadores, que utilizaram o HONcode $(4,11,12)$.

As pessoas com deficiência ou com doença crônica estão mais propensas a usar a internet para acessar sites com informações de saúde. Além da busca por informações, as pessoas que buscam informações, desejam compartilhar suas aflições e experiências.

$\mathrm{O}$ acesso a página do MieloFórum foi disponibilizado ao público a partir do dia 22 de novembro de 2014, havendo um baixo número de acessos devido a contínua manutenção para aperfeiçoamento do mesmo e falta de divulgação até o dia 28 de fevereiro de 2014, quando foi criada a página oficial do MieloFórum na página de relacionamentos da web mais utilizada atualmente, o Facebook, onde os acessos foram crescendo continuadamente chegando a 1548 acessos e atingindo 50 países diferentes em apenas 5 meses, abrangendo um público variado e de diversas localizações regionais como São Paulo, Belo Horizonte, Rio de Janeiro e Paraná, e internacionais incluindo países como EUA, Alemanha e diversos outros mostrados na Tabela 3, evidenciando a grande demanda de interesses de pacientes com Espinha Bífida. A taxa de rejeição evidenciada em $57,04 \%$ se dá devido ao Fórum atualmente ser disponível apenas em português, e grande número dos acessos foram de nível internacional, trazendo grande dificuldade aos usuários e impedindo a continuação do acesso as informações disponíveis.

Foi observado que 20,03\% de acessos ao MieloFórum foram de dispositivos móveis (celulares ou tablets). 0 número de pessoas que declararam acessar a internet pelo celular cresceu $65 \%$ no último $a{ }^{(13)}$ o que nos mostra uma possível necessidade da criação de um aplicativo para facilitar o acesso por redes móveis.

\section{CONCLUSÃO}

O desenvolvimento de uma ferramenta que permita a divulgação de informações sobre saúde de qualidade e a troca de experiências entre os usuários, como um fórum virtual, é sempre um desafio. Especialmente quando essa ferramenta é destinada a um público com limitações físicas e cognitivas, como os indivíduos com Espinha Bífida.

Nessa direção a análise da acessibilidade, com o AChecker, foi essencial para garantir e facilitar o acesso do público alvo desse fórum virtual de saúde, além de indicar a necessidade de transferência da plataforma utilizada. A certificação pelo HONcode elevou o
MieloFórum a uma padronização internacional, visando a transparência e clareza das informações de saúde, proporcionando ao usuário uma segurança ao utilizar o fórum.

Seguindo a ideia de inovação e novas tecnologias de informação, uma ferramenta como o MieloFórum tem muito a crescer, necessitando de manutenção e aperfeiçoamento contínuos para esclarecer e oferecer informações sobre saúde de qualidade aos usuários. Devido os acessos provenientes de outros países, foi observada a grande relevância de disponibilizar uma versão em inglês, visando aumentar o acesso e diminuir a taxa de rejeição de usuários que não falam a língua portuguesa.

O fórum é um espaço privilegiado para discussões e debates, é uma ferramenta de comunicação muito versátil. É um espaço onde todos podem ver o que todos fazem, ainda que não ao mesmo tempo. No processo de ensino e de aprendizagem os benefícios consistem no processo de meta cognição onde o participante ao expressar suas ideias, precisa organizá-las de forma clara e objetiva e por fim fazer análise do que aprendeu, torna-se assim um banco de informações. A participação nos fóruns nos possibilita aprender, ensinar e estabelecer relacionamentos com outras pessoas $^{(10)}$.

O desenvolvimento de um fórum é sempre um desafio, e o alto número de acessos mostra a importância e a demanda de trazer as pessoas com espinha bífida uma ferramenta virtual que possibilite o esclarecimento de dúvidas sobre saúde e a oportunidade de interação, bem como o compartilhamento de experiências. 0 MieloFórum mostrou-se não apenas para uma fonte de informação sobre saúde, mas também uma rede de apoio mútuo para essa população.

\section{REFERÊNCIAS BIBLIOGRÁFICAS}

1. AChecker. Web Accessibility Checker 2013. Available from: http: //achecker.ca/checker/index.php.

2. HON. Health on the Net Foundation 2013 [cited 2013]. Available from: http://www.hon.ch/.

3. W3C. The World Wide Web Consortium 2013. Disponível em: <http://www.w3.org/>.Whereoware. Disponível em: < http: //www. whereoware.com/blog/not-set-vs-notprovided/2013/05/>

4. Amaral Lad. Um ambiente para análise de resultados de avaliações de acessibilidade e usabilidade na Web. São Carlos: Universidade de São Paulo; 2014.

5. Ipser JC, Dewing S, Stein DJ. A systematic review of the quality of information on the treatment of anxiety disorders on the internet. Curr Psychiatry Rep. 2007;9(4):303-9.

6. Boyer C, Gaudinat A, Baujard V, and Geissbuhler A. Health on the Net Foundation: assessing the quality of health web pages all over the world. Stud Health Technol Inform. 2007; 129(Pt 2):1017-21.

7. Vanti, N. Indicadores web e sua aplicação à produção científica disponibilizada em revistas eletrônicas. In: Ferreira, S. M. S. P.; Targino, M. G. (Org.). Acessibilidade e visibilidade de revistas científicas eletrônicas. São Paulo: Ed. SENAC, 2010. p. 175-212.

8. Varela RCB, Oliver FC. A utilização da tecnologia assistiva na vida de crianças com deficiências. Cien Saúde Coletiva [Internet]. 2013 [acesso 2018 Out 12]; 18 ${ }^{(6)}: 1773-84$. Disponível em: https: // dx.doi.org/10.1590/S1413-81232013000600028

9. Google Analytics. Disponível em: <http://www.google.com/analytics/>. Acesso em: 17/07/2015.

10. Oh YS, Cho Y. Examining the relationschips between resources 
and online helth information seeking among patients with chronic diseases and healthy people. Soc Work Health Care [Internet]. 2015 Fev. [acesso 2018 Out 12]; 54(2):83-100. Disponível em: https://doi.org/10.10 80/00981389.2014.987940

11. Mendonça APB, Pereira-Neto A. Critérios de avaliação da qualidade da informação em sites de saúde: uma proposta. Rev Eletr Comun Inf Inov Saúde [Internet]. 2015 Mar. [acesso 2018 Out 12]; $\quad 9^{(1)}: 1-15$. Disponível em: https: / /dx.doi.org/10.29397/reciis.v9i1.930

12. Fagnano M, Halterman JS, Conn KM, Shone LP. Health literacy and sources of health information for caregivers of urban children with asthma. Clin Pediatr (Phila). 2012; 51(3):267-73.

13. Pesquisa de Mídia Brasileira. Disponível em: <http://www.secom.gov.br/atuacao/pesquisa/lista-depesquisas-quantitativas-e-qualitativas-de-contratos-

atuais/pesquisa-brasileira-de-midia-pbm-2015.pdf. Acesso em: $10 / 07 / 2015>$. 